\title{
Estado de la susceptibilidad de poblaciones naturales del vector del dengue a insecticidas en trece localidades de Colombia
}

\author{
Liliana Santacoloma', Bernardo Chaves², Helena Luisa Brochero² \\ ${ }^{1}$ Laboratorio de Entomología, Subdirección Red Nacional de Laboratorios, Instituto Nacional de Salud, Bogotá, \\ D.C., Colombia \\ 2 Facultad de Agronomía, Universidad Nacional de Colombia, Bogotá, D.C., Colombia \\ Institución en la que se llevó a cabo el trabajo: Laboratorio de Entomología, Instituto Nacional de Salud, Bogotá, \\ D.C., Colombia.
}

Introducción. La resistencia fisiológica de las poblaciones naturales de Aedes aegypti a los insecticidas de uso en salud pública, disminuye la eficacia del control químico utilizado para interrumpir la transmisión de dengue durante los brotes y epidemias.

Objetivo. Determinar el estado de la susceptibilidad al carbamato propoxur, a los organofosforados adulticidas malatión y fenitrotión y al larvicida temefos, de 13 poblaciones naturales de Ae. aegypti en ocho municipios: una de Bucaramanga y dos de Sabana de Torres en el departamento de Santander; dos de Girardot y dos de La Mesa en Cundinamarca; dos de Villavicencio y dos de Puerto López en el Meta; una de San José del Guaviare, en Guaviare, y una de Florencia en Caquetá.

Materiales y métodos. Las pruebas de susceptibilidad consistieron en bioensayos estandarizados de la Organización Mundial de la Salud (OMS), 1981, y botellas de los Centers for Disease Control and Prevention (CDC), 1998. Se aplicaron pruebas colorimétricas para determinar los niveles de enzimas como posibles responsables de la reducción de la susceptibilidad a insecticidas organofosforados y al carbamato propoxur.

Resultados. Todas las poblaciones presentaron susceptibilidad a malatión y propoxur. Para el temefos, solo cuatro de las trece localidades evaluadas registraron susceptibilidad. Con respecto a los posibles mecanismos de desintoxicación enzimática, en siete de las once poblaciones naturales se registraron valores superiores al punto de corte para esterasas inespecíficas, solamente en Bucaramanga se presentó un incremento en las enzimas monooxigenasas del citocromo P450, pero en ninguna población se registró acetilcolinestarasa modificada.

Conclusiones. La susceptibilidad generalizada a los organofosforados adulticidas evaluados, indica que el malatión, el insecticida más utilizado en Colombia para interrumpir la transmisión del dengue, sigue siendo eficaz. Las particularidades de los resultados de la resistencia fisiológica a insecticidas de las comunas de un mismo municipio, evidenciaron un fenómeno localizado de manera específica en espacio y tiempo.

Palabras clave: control de vectores, Aedes, dengue, resistencia a insecticidas, insecticidas organofosforados, carbamatos.

doi: http://dx.doi.org/10.7705/biomedica.v32i3.680

\section{Susceptibility of natural populations of dengue vector to insecticides in Colombia}

Introduction. Physiological resistance of natural population of Aedes aegypti to insecticides contribute to the decreased efficacy of chemical control as a main control strategy during dengue outbreaks.

Objective. The susceptibility status of Ae. aegypti was assessed for the carbamate propoxur, the adulticide malathion and the larvicide temephos on 13 natural populations of Ae. aegypti immature forms were taken from 8 Colombian localities. These included the following: Bucaramanga (1), Sabana de Torres (2), Girardot (2), La Mesa (2), Villavicencio (2), Puerto López (2), San José del Guaviare (1) and Florencia (1).

Materials and methods. Susceptibility tests mainly consisted of the standardized bioassay outlined by WHO (1981) and CDC bottles (1998). Colorimetric tests were undertaken to determine enzyme levels possibly responsible for the reduction of susceptibility to organophosphate and carbamate insecticides.

\section{Contribución de los autores:}

Liliana Santacoloma: formulación del proyecto de investigación, realización del trabajo y redacción del artículo.

Bernardo Chaves: asesoría en el análisis estadístico de los datos y sugerencias al artículo.

Helena Brochero: dirección del proyecto de investigación y corrección del artículo. 
Results. All specimens demonstrated susceptibility to malathion and propoxur insecticides. Four of the 13 populations revealed susceptibility to the temephos larvicide. Seven of 11 populations showed a limited increase in values for nonspecific esterase enzymes. The Bucaramanga population was the only one which showed an increase in the cytochrome P450 monooxygenases enzymes. Neither population was found with modified acetilcolinesterase.

Conclusions. The widespread susceptibility to organophosphates used as adulticides indicated that malathion, the most used insecticide in Colombia, remains effective in interrupting the transmission of dengue. Physiological resistance to insecticides occurring in communities of a single township proved to be a localized phenomenon.

Key words: Vector control, Aedes, dengue, insecticide resistance; insecticides, organophosphate; carbamates.

doi: http://dx.doi.org/10.7705/biomedica.v32i3.680

El dengue y su forma grave constituyen la enfermedad viral de transmisión vectorial más importante en los países tropicales y subtropicales (1). En América, y en Colombia, el número de casos graves y muertes por dengue ha aumentado en los últimos años (2). Durante 2001 a 2007, murieron 222 personas en Colombia a causa del dengue, lo que representó el $75 \%$ de la mortalidad ocasionada por esta enfermedad para el conjunto de los países andinos (3) y, solo en el 2010, durante la epidemia de dengue más grave que ha experimentado el país, el número de muertes confirmadas fue de 217 (4).

Ante la ausencia de una vacuna que proteja la población en riesgo, el control vectorial es la medida más importante para la prevención e interrupción de la transmisión en caso de epidemia (5). Como medidas de prevención, se realizan campañas educativas dirigidas a la comunidad, con el fin de eliminar los criaderos del vector, lo cual se complementa con la aplicación de larvicidas en criaderos de difícil eliminación (3) y depósitos de agua potable de uso doméstico. Los larvicidas autorizados por el Programa Nacional de Enfermedades Transmitidas por Vectores del Ministerio de Salud y Protección Social para el control focal, son la bacteria Bacillus thurigiensis variedad israelensis (6), los reguladores de crecimiento y el organofosforado temefos; este último ha sido el más utilizado en el país durante los últimos años dada su mayor capacidad residual y compatibilidad con los programas de control social. En los casos de epidemia, las poblaciones

\section{Correspondencia:}

Liliana Santacoloma, Laboratorio de Entomología, Instituto Nacional de Salud, Avenida calle 26 № 51-60, Bogotá, D.C., Colombia

Teléfono: 220 7700, extensiones 1342 y 1343

lisantacoloma@gmail.com, Isantacoloma@ins.gov.co

Recibido: 15/03/11; aceptado:15/03/12 de adultos se controlan mediante aspersiones con insecticidas químicos. El organofosforado malatión ha sido la molécula más utilizada en el país desde los años ochenta (7), seguido en menor proporción, por los piretroides lambdacialotrina y deltametrina.

La aparición de resistencia es una de las causas del fracaso del control de vectores. En Colombia, el hallazgo en 1956 de resistencia al DDT en una población de Aedes aegypti Linnaeus 1762 de Cúcuta (Norte de Santander) (8) y la consecuente reinfestación por el vector, conllevaron a la utilización de los organosforados, como el larvicida temefos y, posteriormente, el adulticida malatión (9). Sin embargo, desde 1990, año del primer reporte de resistencia al temefos en Cali (Suárez M, González R, Morales C. Temephos resistance to Aedes aegypti in Cali, Colombia. Am J Trop Med Hyg. 1996;55(Suppl.):257), la detección de resistencia a esta molécula en diferentes estudios con poblaciones de Ae. aegypti de campo ha sido frecuente $(7,9)$ (González J, Rey G, Olano $\mathrm{V}$, Brochero $\mathrm{H}$. Informe técnico, Ministerio de la Protección Social. 2005; Convenio 043. p. 60). Los registros de resistencia al malatión, adulticida más utilizado en el control de Ae. aegypti en Colombia, son escasos. Con el primero de ellos, en 1995 en una población de Antioquia (10), y posteriormente en el 2003, se evidenció pérdida de susceptibilidad en una población de Barrancabermeja (Santander) (Rojas W, Gónzález J, Amud M, Quiñones M, Vélez I. Evaluación de la susceptibilidad de Aedes aegypti del municipio de Barrancabermeja, Santander a los insecticidas malatión, fenitrotión, temefos, lambdacialotrina, deltametrina, permetrina, propoxur y DDT. 2003. Biomédica. 2003;23(Supl.1):35-6.).

Teniendo en cuenta la necesidad de contar con una línea base de susceptibilidad de Ae. aegypti a los insecticidas de uso en salud pública y establecer un programa de vigilancia de la resistencia a 
insecticidas que aporte información para la toma de decisiones relacionadas con las medidas de control, durante el 2005 al 2007 se llevó a cabo el proyecto: "Evaluación del estado actual de la resistencia a insecticidas en los principales vectores de malaria, dengue y fiebre amarilla urbana en Colombia e iniciación de la Red Nacional de Vigilancia de la Resistencia a Insecticidas en Colombia". En este proyecto participaron el Centro de Entrenamiento e Investigaciones Médicas (CIDEIM), el Programa de Estudio y Control de Enfermedades Tropicales de la Universidad de Antioquia (PECET), el Instituto Nacional de Salud y la Universidad Nacional de Colombia, con el apoyo de las secretarías departamentales de salud y la financiación de Colciencias.

Los resultados que aquí se presentan hacen parte de una serie de estudios del Instituto Nacional de Salud en trece localidades seleccionadas en el marco de dicho proyecto.

\section{Materiales y métodos}

\section{Sitios de estudio}

En cada uno de los cinco departamentos de Colombia incluidos en el estudio, se seleccionaron uno o dos municipios con mayor incidencia de dengue. En cada municipio se escogieron dos localidades o "comunas", de acuerdo con historia de índices de Breteau superiores a 5 y el registro sistemático de intervenciones químicas para el control de casos de dengue. De cada comuna seleccionada, que consistió en una agrupación de barrios con características socio-demográficas similares, se recolectaron las 13 poblaciones naturales de Ae. aegypti evaluadas. En el cuadro 1 se registran los sitios de estudio.

\section{Material biológico}

En cada comuna se recolectaron larvas de Ae. aegypti siguiendo la metodología estandarizada por la Organización Panamericana de la Salud (OPS) para el levantamiento de índices de infestación aédica (11). El material de campo se llevó al laboratorio y se mantuvo en condiciones controladas con una temperatura de $24 \pm 4{ }^{\circ} \mathrm{C}, 70 \%$ de humedad y un fotoperiodo de 12:12 horas. Las larvas se dispusieron en bandejas plásticas de $30 \times 30 \mathrm{~cm}$ con agua sin cloro y se alimentaron diariamente con Rodentina ${ }^{\circledR}$ pulverizada para obtener adultos, los cuales se alimentaron con solución azucarada al $10 \%$; para facilitar la postura de huevos, las hembras se alimentaron cada tercer día con sangre de ratón de la especie Mus muscullus, siguiendo los protocolos de manejo de vertebrados del Instituto Nacional de Salud. Una fracción de hembras F1, aproximadamente 200 individuos, se almacenaron a $-70^{\circ} \mathrm{C}$ a los dos días de emergidas, con el propósito de estandarizar la edad fisiológica y utilizarlas para la evaluación de los posibles mecanismos de desintoxicación enzimática asociados con resistencia. A partir de las hembras F1 restantes y los machos de esta generación, se obtuvo la cantidad suficiente de hembras $\mathrm{F} 2$ de tres a seis días de nacidas utilizadas en los bioensayos.

Cuadro 1. Origen geográfico a partir del cual se obtuvieron las poblaciones naturales de Aedes aegypti evaluadas

\begin{tabular}{|c|c|c|c|c|}
\hline Departamento & Municipio & Coordenadas & Altitud (msnm) & Comunas evaluadas \\
\hline Caquetá & Florencia & $\begin{array}{l}01^{\circ} 36^{\prime} 59^{\prime \prime} \mathrm{N} \\
75^{\circ} 36^{\prime} 00^{\prime \prime} \mathrm{W}\end{array}$ & 242 & Florencia \\
\hline \multirow[t]{2}{*}{ Cundinamarca } & Girardot & $\begin{array}{l}04^{\circ} 18^{\prime} 11^{\prime \prime} \mathrm{N} \\
74^{\circ} 48^{\prime} 03^{\prime \prime} \mathrm{W}\end{array}$ & 289 & $\begin{array}{l}\text { Girardot } 1 \\
\text { Girardot } 2\end{array}$ \\
\hline & La Mesa & $\begin{array}{l}04^{\circ} 38^{\prime} 09^{\prime \prime} \mathrm{N} \\
74^{\circ} 27^{\prime} 59^{\prime \prime} \mathrm{W}\end{array}$ & 1300 & $\begin{array}{l}\text { La Mesa } 1 \\
\text { La Mesa } 2\end{array}$ \\
\hline Guaviare & San José del Guaviare & $\begin{array}{l}02^{\circ} 34^{\prime} 37^{\prime \prime} \mathrm{N} \\
72^{\circ} 38^{\prime} 45^{\prime \prime} \mathrm{W}\end{array}$ & 200 & San José del Guaviare \\
\hline \multirow[t]{2}{*}{ Meta } & Villavicencio & $\begin{array}{l}04^{\circ} 09^{\prime} 12^{\prime \prime} \mathrm{N} \\
73^{\circ} 38^{\prime} 06^{\prime \prime} \mathrm{W}\end{array}$ & 457 & $\begin{array}{l}\text { Villavicencio } 7 \\
\text { Villavicencio } 5\end{array}$ \\
\hline & Puerto López & $\begin{array}{l}04^{\circ} 05^{\prime} 06^{\prime \prime} \mathrm{N} \\
72^{\circ} 57^{\prime} 19^{\prime \prime} \mathrm{W}\end{array}$ & 184 & $\begin{array}{l}\text { Puerto López } 1 \\
\text { Puerto López } 2\end{array}$ \\
\hline \multirow[t]{2}{*}{ Santander } & Bucaramanga & $\begin{array}{l}07^{\circ} 07^{\prime} 47^{\prime \prime} \mathrm{N} \\
73^{\circ} 07^{\prime} 33^{\prime \prime} \mathrm{W}\end{array}$ & 960 & Bucaramanga 17 \\
\hline & Sabana de Torres & $\begin{array}{l}07^{\circ} 23^{\prime} 53^{\prime \prime} \mathrm{N} \\
73^{\circ} 29^{\prime} 49^{\prime \prime} \mathrm{W}\end{array}$ & 234 & $\begin{array}{l}\text { Sabana de Torres } 1 \\
\text { Sabana de Torres } 2\end{array}$ \\
\hline
\end{tabular}




\section{Determinación de la susceptibilidad en larvas}

Para la determinación de la susceptibilidad al larvicida organofosforado temefos, se utilizó insecticida de grado técnico (99\%) adquirido en Chem Service (West Chester, PA, USA). Se aplicó la metodología de la OMS (12), evaluando la dosis diagnóstica obtenida por el PECET en el marco del proyecto nacional de resistencia a insecticidas, la cual correspondió a 0,012 ppm (13). Cada prueba consistió de cuatro réplicas y un control. Cada réplica, así como los controles, consistió en un vaso de vidrio provisto con $99 \mathrm{ml}$ de agua sin cloro. A cada uno de los vasos con los tratamientos se les agregó $1 \mathrm{ml}$ de temefos a una concentración de 1,2 ppm, para obtener una concentración final de 0,012 ppm. A los vasos control se les agregó $1 \mathrm{ml}$ de etanol absoluto libre de insecticida. Se evaluaron 25 larvas entre el tercer estadio tardío y el cuarto temprano por réplica, para un total de 100 larvas por prueba. Se hicieron tres pruebas por población del insecto evaluado. La lectura de mortalidad se hizo a las 24 horas. Se consideraron larvas muertas aquellas con: 1) ausencia de respuesta al ser estimuladas dorsalmente con una micropinza, 2) incapacidad de ascender a la superficie y de llegar al fondo del vaso, y 3) con presencia de necrosis de la epidermis (12).

\section{Determinación de la susceptibilidad en mosquitos adultos}

Para las evaluaciones de sensibilidad de las hembras adultas se utilizaron dos metodologías estandarizadas, correspondientes a papeles impregnados de la OMS, 1981 (14), y botellas impregnadas de los Centers for Disease Control and Pravention (CDC), 1998 (15). Tanto para la metodología de la OMS como la de los CDC, se hicieron tres pruebas por cada insecticida. Cada prueba estuvo conformada por cuatro réplicas del tratamiento y un control. Una réplica consistió en un tubo (OMS) o botella (CDC), dentro del cual se colocaron entre 20 y 25 hembras del mosquito F2 entre tres y seis días de nacidas y alimentadas con agua azucarada al $10 \%$.

En los bioensayos OMS se utilizaron papeles impregnados a una concentración de 0,1\% para propoxur, de 5,0 \% para malatión y de 1,0\% para fenitrotión. Los controles consistieron en papeles impregnados con aceite de oliva, solvente utilizado para estos principios activos. Los papeles impregnados y los controles para los bioensayos OMS, fueron adquiridos en Malasia, de acuerdo con las recomendaciones de la Organización
Mundial de la Salud (14). El criterio de mortalidad consistió en la evaluación de individuos evidentemente muertos a las 24 horas después de la exposición (14). Los bioensayos CDC requirieron de la determinación previa de los tiempos y dosis diagnósticas para cada molécula insecticida evaluada, los cuales se obtuvieron con la cepa de referencia Rockefeller por el PECET de la Universidad de Antioquia (13). Las dosis diagnósticas utilizadas en los bioensayos CDC correspondieron a $12,5 \mu \mathrm{g} / \mathrm{ml}$ por 30 minutos para el carbamato propoxur, a $100 \mu \mathrm{g} / \mathrm{ml}$ por 30 minutos para malatión y a $75 \mu \mathrm{g} / \mathrm{ml}$ por 45 minutos para fenitrotión (13). Las botellas control se impregnaron con etanol absoluto. El criterio de mortalidad consistió en mosquitos evidentemente muertos o con síntomas de intoxicación, como incapacidad de volar o posarse después de caer sobre el dorso (15). Los insecticidas de grado técnico propoxur (99\%), malatión (98\%) y fenitrotión (99,5\%), utilizados tanto en la obtención de la dosis diagnóstica como en las pruebas de sensibilidad, se adquirieron en Chem Service (West Chester, PA, USA). Todos los bioensayos se llevaron a cabo a una temperatura ambiental de 18 a $20{ }^{\circ} \mathrm{C}$ y una humedad relativa de 65 a $68 \%$.

\section{Análisis de resultados}

Tanto para los bioensayos OMS (en adultos y larvas) como en los bioensayos CDC con adultos, se obtuvieron los promedios de las mortalidades, expresadas en porcentajes, de las tres pruebas por insecticida. Cuando en una réplica control de una prueba se presentaron mortalidades inferiores a $20 \%$, los resultados de la mortalidad total se corrigieron utilizando la fórmula de Schneider y Orelli (16). Los bioensayos que presentaron mortalidad superior a este porcentaje se descartaron y la prueba se repitió. Para los resultados con la metodología CDC (1998), la supervivencia al tiempo diagnóstico se consideró como pérdida de sensibilidad al insecticida evaluado. En el caso de la metodología OMS (1981), las poblaciones con más de $98 \%$ de mortalidad se consideraron susceptibles, aquellas con menos de $80 \%$, resistentes, y en aquellas con 80 a $98 \%$, se consideraron valores de vigilancia para los cuales se debe corroborar la presencia o ausencia de resistencia. Teniendo en cuenta que cada población se evaluó con ambas metodologías, OMS y CDC, para cada insecticida, los criterios de susceptibilidad se unificaron en los siguientes casos: 1) poblaciones resistentes, aquellas con pérdida de susceptibilidad en CDC y 
resistencia en OMS, y 2) poblaciones con pérdida de susceptibilidad, las cuales presentaron pérdida de susceptibilidad en CDC y vigilancia en OMS.

\section{Determinación de los mecanismos enzimáticos de resistencia}

Con el propósito de determinar alteraciones de tipo enzimático asociadas con la resistencia fisiológica a los insecticidas organofosforados y el carbamato propoxur en las poblaciones evaluadas, se aplicaron pruebas colorimétricas de acuerdo con la metodología propuesta (17-19). Utilizando un lector para la ELISA (Thermo Labsystems), se midieron las densidades ópticas a longitudes de onda predeterminadas para determinar el incremento en el nivel de las enzimas esterasas inespecíficas y monooxigenasas del grupo citocromo P450 (CIP450).

Para la acetilcolinesterasa modificada, se determinó la proporción de individuos de la población que no presentaban inhibición de la enzima acetilcolinesterasa. El incremento de las enzimas esterasas inespecíficas, se determinó utilizando éster $\beta$-naphtil acetato (Merck) como sustrato, $\beta$-naphtol (Merck) como control positivo, y la solución amortiguadora fosfato como control negativo. Después de 10 minutos de incubación, se agregó dianisidina (Sigma), compuesto que evidencia la formación de $\beta$-naphtol. Se leyó la absorbancia utilizando el filtro de $570 \mathrm{~nm}$.

Para determinar el incremento de las monooxigenasas del grupo citocromo P450, se utilizó peróxido de hidrógeno (Microgen Chemicals) como sustrato, citocromo C (Merck) como control positivo, solución amortiguadora fosfato como control negativo y 3.3', 5,5'tetrametil-bencidina-TMBZ (Sigma) que evidencia la presencia de peroxidasas y peróxido de hidrogeno por oxidación. Se leyó la absorbancia utilizando al tiempo 0 y 10 y el filtro de $630 \mathrm{~nm}$.

La determinación de acetilcolinesterasa modificada consistió en utilizar una mezcla de acetilcolina (Sigma) más propoxur (Chem Service) como sustrato, acetilcolina iodada (Sigma) como control positivo, solución amortiguadora fosfato como control negativo y DTNB (5,5'ditio-bis, 2-ácido nitrobenzoico) (Sigma) compuesto que reacciona con la tiocolina, la cual es producto de la hidrólisis de la acetilcolina efectuada por la enzima colinesterasa. Se leyó la absorbancia al tiempo 0 y 10 utilizando el filtro de $410 \mathrm{~nm}$.

En el caso particular de la CIP450 y la acetilcolinesterasa modificada, se restó el valor de absorbancia del tiempo 0 al valor de absorbancia del tiempo 10 para cada una de las muestras. Para determinar el incremento en el nivel de enzimas esterasas inespecíficas y CIP450, y el número de individuos con acetilcolinesterasa modificada, se establecieron puntos de corte con la cepa de referencia Rockefeller de Ae. aegypti (18). Los promedios de las densidades ópticas de las poblaciones de campo iguales o inferiores a este valor se consideraron como normales, y los valores superiores, como incrementos en el nivel de las enzimas esterasas inespecíficas y CIP450 o presencia de acetilcolinesterasa modificada.

Para el análisis estadístico de los datos se aplicaron las pruebas de: 1) Shapiro-Wilk, intervalo de confianza $\left(\mathrm{IC}_{95 \%}\right)$, para determinar la normalidad; 2) análisis de varianza $\left(\mathrm{IC}_{95 \%}\right)$ para comparar las densidades ópticas entre poblaciones; 3 ) la prueba de comparación múltiple de Dunnett $\left(\mathrm{IC}_{95 \%}\right)$ para comparar las densidades ópticas entre poblaciones y Rockefeller (18).

Los promedios de mortalidad para los resultados de las pruebas biológicas, así como el análisis de resultados de las pruebas bioquímicas, se obtuvieron con el programa estadístico $\mathrm{SAS}^{\circledR}$, versión 9,1 (SAS Institute, Cary, NC, USA).

\section{Resultados}

\section{Determinación de la susceptibilidad}

En el cuadro 2 se registra el promedio aritmético de los porcentajes de mortalidad de Ae. aegypti de 13 comunas correspondientes a cinco departamentos del centro-oriente colombiano, evaluadas para los insecticidas malatión, fenitrotión, propoxur y el larvicida temefos.

Todas las poblaciones naturales de Ae. aegypti presentaron susceptibilidad a malatión, tanto en los bioensayos OMS como en los CDC. Para el propoxur, todas las poblaciones presentaron susceptibilidad con la metodología CDC, en tanto que los valores de mortalidad con OMS fueron muy bajos en todas las poblaciones, incluyendo la cepa de referencia Rockefeller (no se presentan los datos). Asumiendo un posible problema de estabilidad del compuesto en los papeles impregnados OMS, solo se discuten los resultados obtenidos mediante bioensayos CDC. Para el organofosforado fenitrotión, todas las poblaciones mostraron susceptibilidad usando CDC, en tanto que para OMS, $4 / 13$ poblaciones correspondientes a la Mesa 1, Puerto López 1 y 2 y Sabana de Torres, evidenciaron porcentajes de mortalidad 
Cuadro 2. Mortalidad de Aedes aegypti en 13 localidades evaluadas para dosis diagnósticas de insecticidas adulticidas y larvicidas insecticidas

\begin{tabular}{|c|c|c|c|c|c|c|c|c|c|c|c|c|c|c|c|}
\hline \multirow[b]{3}{*}{ Deptos. } & \multirow[b]{3}{*}{ Comunas } & \multicolumn{4}{|c|}{$\begin{array}{c}\text { Malatión } \\
\text { Adulticida } \\
\text { organosfosforado }\end{array}$} & \multicolumn{4}{|c|}{$\begin{array}{c}\text { Fenitrotión } \\
\text { Adulticida } \\
\text { organosfosforado }\end{array}$} & \multirow{2}{*}{\multicolumn{2}{|c|}{$\begin{array}{c}\begin{array}{c}\text { Propoxur } \\
\text { Adulticida } \\
\text { carbamato }\end{array} \\
\text { OMS }\end{array}$}} & \multicolumn{4}{|c|}{$\begin{array}{c}\text { Temefos } \\
\text { Larvicida } \\
\text { organofosforado }\end{array}$} \\
\hline & & \multicolumn{2}{|c|}{$\begin{array}{l}\text { Metodologías } \\
\text { OMS }\end{array}$} & \multicolumn{2}{|c|}{ CDC } & \multicolumn{2}{|c|}{ OMS } & \multicolumn{2}{|c|}{ CDC } & & & \multicolumn{2}{|c|}{ CDC } & \multicolumn{2}{|c|}{ OMS } \\
\hline & & $\%$ & $\mathrm{DE}$ & $\%$ & DE & $\%$ & $\mathrm{DE}$ & $\%$ & $\mathrm{DE}$ & $\%$ & $\mathrm{DE}$ & $\%$ & $\mathrm{DE}$ & $\%$ & SD \\
\hline \multirow[t]{4}{*}{ Cundinamarca } & Girardot 1 & $\begin{array}{c}100 \\
n=300\end{array}$ & $\pm 0,0$ & $\begin{array}{c}100 \\
n=175\end{array}$ & $\pm 0,0$ & $\begin{array}{c}90 \\
\mathrm{n}=100\end{array}$ & $\pm 1,9$ & $\begin{array}{c}100 \\
n=100\end{array}$ & $\pm 0,0$ & $\begin{array}{c}15 \\
n=100\end{array}$ & $\pm 7,6$ & $\begin{array}{c}100 \\
n=100\end{array}$ & $\pm 0,0$ & $\begin{array}{c}48 \\
n=160\end{array}$ & $\pm 2,3$ \\
\hline & Girardot 3 & $\begin{array}{c}100 \\
n=300\end{array}$ & $\begin{array}{c} \pm 0,0 \\
\mathrm{n}=100\end{array}$ & 100 & $\begin{array}{c} \pm 0,0 \\
\mathrm{n}=100\end{array}$ & 91 & $\begin{array}{c} \pm 1,6 \\
\mathrm{n}=100\end{array}$ & 100 & $\begin{array}{c} \pm 0,0 \\
\mathrm{n}=100\end{array}$ & 26 & $\begin{array}{l} \pm 19,5 \\
n=112\end{array}$ & 100 & $\begin{array}{c} \pm 0,0 \\
\mathrm{n}=160\end{array}$ & $\begin{array}{c}54 \\
\pm 3,8\end{array}$ & \\
\hline & La Mesa 1 & $\begin{array}{c}100 \\
n=100\end{array}$ & $\pm 0,0$ & $\begin{array}{c}100 \\
n=100\end{array}$ & $\pm 0,0$ & $\begin{array}{c}14 \\
n=100\end{array}$ & $\pm 8,4$ & $\begin{array}{c}100 \\
\mathrm{n}=181\end{array}$ & $\pm 0,0$ & $\begin{array}{c}56 \\
n=100\end{array}$ & $\pm 5,7$ & $\begin{array}{c}100 \\
n=191\end{array}$ & $\pm 0,0$ & $\begin{array}{c}23 \\
n=240\end{array}$ & $\pm 9,5$ \\
\hline & La Mesa 2 & $\begin{array}{c}100 \\
n=124\end{array}$ & $\pm 0,0$ & $\begin{array}{c}85 \\
n=100\end{array}$ & $\pm 6,9$ & $\begin{array}{c}96 \\
n=100\end{array}$ & $\pm 5,2$ & $\begin{array}{c}100 \\
n=120\end{array}$ & $\pm 0,0$ & $\begin{array}{c}56 \\
\mathrm{n}=158\end{array}$ & $\pm 4,8$ & $\begin{array}{c}100 \\
n=100\end{array}$ & $\pm 0,0$ & $\begin{array}{c}1,2 \\
n=240\end{array}$ & $\pm 2,2$ \\
\hline Caquetá & Florencia & $\begin{array}{c}98 \\
n=117\end{array}$ & $\pm 4,0$ & $\begin{array}{c}100 \\
n=159\end{array}$ & $\pm 0,0$ & $\begin{array}{c}100 \\
n=293\end{array}$ & $\pm 0,0$ & $\begin{array}{c}100 \\
n=120\end{array}$ & $\pm 0,0$ & $\begin{array}{c}57 \\
n=100\end{array}$ & $\pm 18,0$ & $\begin{array}{c}100 \\
n=100\end{array}$ & $\pm 0,0$ & --- & --- \\
\hline Guaviare & San José & $\begin{array}{c}98 \\
\mathrm{n}=119\end{array}$ & $\pm 3,7$ & $\begin{array}{c}100 \\
n=181\end{array}$ & $\pm 0,0$ & $\begin{array}{c}95 \\
n=100\end{array}$ & $\pm 4,9$ & $\begin{array}{c}100 \\
n=185\end{array}$ & $\pm 0,0$ & $\begin{array}{c}1 \\
n=100\end{array}$ & $\pm 1,7$ & $\begin{array}{c}95 \\
\mathrm{n}=101\end{array}$ & $\pm 5,0$ & $\begin{array}{c}40 \\
n=200\end{array}$ & $\pm 4,0$ \\
\hline \multirow[t]{2}{*}{ Meta } & Villavicencio 5 & $\begin{array}{c}99 \\
n=100\end{array}$ & $\pm 2,2$ & $\begin{array}{c}100 \\
n=100\end{array}$ & $\pm 0,0$ & $\begin{array}{c}95 \\
n=100\end{array}$ & $\pm 5,4$ & $\begin{array}{c}100 \\
n=100\end{array}$ & $\pm 0,0$ & $\begin{array}{c}3 \\
n=100\end{array}$ & $\pm 5,7$ & $\begin{array}{c}100 \\
n=100\end{array}$ & $\pm 0,0$ & $\begin{array}{c}67 \\
n=240\end{array}$ & $\pm 1,6$ \\
\hline & Villavicencio 7 & $\begin{array}{c}100 \\
n=100\end{array}$ & $\pm 0,0$ & $\begin{array}{c}100 \\
n=100\end{array}$ & $\pm 0,0$ & $\begin{array}{c}90 \\
n=100\end{array}$ & $\pm 4,5$ & $\begin{array}{c}100 \\
n=100\end{array}$ & $\pm 0,0$ & $\begin{array}{c}4 \\
n=100\end{array}$ & $\pm 3,4$ & $\begin{array}{c}100 \\
n=100\end{array}$ & $\pm 0,0$ & $\begin{array}{c}84 \\
n=240\end{array}$ & $\pm 1,2$ \\
\hline \multirow[t]{5}{*}{ Santander } & Puerto López 1 & $\begin{array}{c}97 \\
\mathrm{n}=179\end{array}$ & $\pm 4,8$ & $\begin{array}{c}100 \\
n=264\end{array}$ & $\pm 0,0$ & $\begin{array}{c}30 \\
\mathrm{n}=179\end{array}$ & $\pm 3,4$ & $\begin{array}{c}100 \\
n=264\end{array}$ & $\pm 0,0$ & $\begin{array}{c}17 \\
\mathrm{n}=160\end{array}$ & $\pm 15,0$ & $\begin{array}{c}100 \\
n=220\end{array}$ & $\pm 0,0$ & $\begin{array}{c}92 \\
n=240\end{array}$ & $\pm 3,4$ \\
\hline & Puerto López 2 & $\begin{array}{c}100 \\
n=227\end{array}$ & $\pm 0,0$ & $\begin{array}{c}100 \\
n=160\end{array}$ & $\pm 0,0$ & $\begin{array}{c}20 \\
\mathrm{n}=227\end{array}$ & $\pm 15,2$ & $\begin{array}{c}100 \\
n=160\end{array}$ & $\pm 0,0$ & $\begin{array}{c}0 \\
n=236\end{array}$ & $\pm 18,0$ & $\begin{array}{c}100 \\
n=233\end{array}$ & $\pm 0,0$ & $\begin{array}{c}98 \\
\mathrm{n}=240\end{array}$ & $\pm 3,4$ \\
\hline & Bucaramanga 17 & $\begin{array}{c}100 \\
n=100\end{array}$ & $\pm 0,0$ & $\begin{array}{c}100 \\
\mathrm{n}=98\end{array}$ & $\pm 0,0$ & $\begin{array}{c}100 \\
n=100\end{array}$ & $\pm 0,0$ & $\begin{array}{c}100 \\
n=100\end{array}$ & $\pm 0,0$ & $\begin{array}{c}8 \\
n=100\end{array}$ & $\pm 8,5$ & $\begin{array}{c}100 \\
n=100\end{array}$ & $\pm 0,0$ & $\begin{array}{c}91 \\
\mathrm{n}=160\end{array}$ & $\pm 6,5$ \\
\hline & $\begin{array}{l}\text { Sabana de } \\
\text { Torres } 1\end{array}$ & $\begin{array}{c}83 \\
n=100\end{array}$ & $\pm 8,4$ & $\begin{array}{c}100 \\
n=100\end{array}$ & $\pm 0,0$ & $\begin{array}{c}91 \\
\mathrm{n}=175\end{array}$ & $\pm 9,5$ & $\begin{array}{c}100 \\
\mathrm{n}=170\end{array}$ & $\pm 0,0$ & $\begin{array}{c}46 \\
n=100\end{array}$ & \pm 10 & $\begin{array}{c}99 \\
\mathrm{n}=100\end{array}$ & $\pm 0,0$ & $\begin{array}{c}36 \\
\mathrm{n}=240\end{array}$ & $\pm 3,0$ \\
\hline & $\begin{array}{l}\text { Sabana de } \\
\text { Torres } 2\end{array}$ & $\begin{array}{c}100 \\
n=100\end{array}$ & $\pm 0,0$ & $\begin{array}{c}95 \\
n=100\end{array}$ & $\pm 8,2$ & $\begin{array}{c}20 \\
n=264\end{array}$ & $\pm 2,0$ & $\begin{array}{c}100 \\
\mathrm{n}=371\end{array}$ & $\pm 0,0$ & $\begin{array}{c}47 \\
n=100\end{array}$ & $\pm 3,0$ & $\begin{array}{c}100 \\
n=100\end{array}$ & $\pm 0,0$ & $\begin{array}{c}56 \\
n=240\end{array}$ & $\pm 2,9$ \\
\hline
\end{tabular}

---: bioensayos no realizados; n: cantidad de individuos evaluados.

OMS: metodología propuesta por la Organización Mundial de la Salud, 1981

CDC: metodología propuesta por los Centers for Disease Control and Prevention (CDC), Atlanta, 1998

demostrativos de resistencia. Para el larvicida temefos, solo 4/12 poblaciones correspondientes a Puerto López 1 y 2, Bucaramanga 17 y Villavicencio 7 registraron valores de susceptibilidad.

\section{Mecanismos enzimáticos de resistencia}

En el cuadro 3 se presentan los valores de las densidades ópticas obtenidos para las tres enzimas evaluadas. Para la acetilcolinesterasa modificada, ninguna de las poblaciones presentó valores superiores al punto de corte de 0,02. Para las enzimas esterasas inespecíficas, 7/11 poblaciones registraron valores de la media de densidad óptica superiores al punto de corte de 0,408: Sabana de Torres 1 con 0,54 registró el valor más alto, representando el $85 \%(51 / 60)$ de los individuos evaluados, y Villavicencio 5 con 0,58 , representando el $81 \%(49 / 60)$ de los individuos evaluados. Para el complejo enzimático de oxidasas de función mixta
CIP450 monooxigenasas, el valor de densidad óptica que representó el punto de corte fue de 0,67 . La población de Ae. aegypti de Bucaramanga 17 registró el $43 \%$ (26/60) de los individuos con valores superiores al punto de corte.

Una vez comprobada la normalidad de los datos, los análisis de varianza ANOVA correspondientes a los valores de las densidades ópticas de las pruebas colorimétricas, mostraron que, en general, todas las enzimas presentaron diferencias significativas entre municipios: acetilcolinesterasa modificada ( $p=0,0009)$, CIP450 $(p<0,0001)$, enzimas esterasas inespecíficas $(p<0,0001)$ y entre comunas acetilcolinesterasa modificada ( $p=0,0262)$, CIP450 $(p<0,0001)$, enzimas esterasas inespecíficas $(p<0,0001)$. Sin embargo, al comparar cada municipio con relación a la cepa de referencia Rockefeller mediante la prueba de rango múltiple de 
Cuadro 3. Puntos de corte, valores promedio de densidades ópticas, desviación estándar y coeficiente de variación para actividad enzimática de: acetil- colinesterasa modificada (ACEM), esterasas inespecíficas (ENE) y oxidasasde función mixta (CIP450), evaluadas para 11 poblaciones naturales de Aedes aegypti y la cepa de referencia Rockefeller, Colombia 20062007.

\begin{tabular}{lccc}
\hline & ACEM & $\begin{array}{c}\text { Enzimas evaluadas } \\
\text { ENE }\end{array}$ & CIP450 \\
\hline Puntos de corte & 0,02 & 0,40 & 0,67 \\
Población & $\bar{X}$ DE CV\% & $\bar{X}$ DE CV\% & DE CV\% \\
Rockefeller & $0,0110,00441$ & $0,2980,0196,4$ & $0,4230,05212,3$ \\
Bucaramanga 17 & $0,0150,00320$ & $0,4500,0132,9$ & $0,7090,0355,0$ \\
Girardot 1 & $0,0250,00315$ & $0,1940,0157,9$ & $0,5670,0427,5$ \\
Girardot 3 & $0,0050,00483$ & $0,2920,0196,4$ & $0,2680,05119,0$ \\
La Mesa 1 & $0,0140,00322$ & $0,4550,0132,9$ & $0,3080,04615,1$ \\
La Mesa 2 & $0,0030,00413$ & $0,2200,0167,3$ & $0,3640,04512,4$ \\
Puerto López 1 & $0,0080,00338$ & $0,4270,0133,1$ & $0,3850,0369,4$ \\
Puerto López 2 & $0,0090,00334$ & $0,2560,0135,0$ & $0,4340,0358,2$ \\
Sabana de Torres 1 & $0,0080,00339$ & $0,5690,0132,3$ & $0,5080,0357,0$ \\
Sabana de Torres 2 & $0,0070,00350$ & $0,4840,0142,8$ & $0,5230,0377,1$ \\
Villavicencio 5 & $0,0100,00329$ & $0,5700,0132,3$ & $0,5070,0357,0$ \\
Villavicencio 7 & $0,0100,00331$ & $0,4190,0143,4$ & $0,6500,0609,2$ \\
\hline
\end{tabular}

X: media; DE: desviación estándar; CV\%: coeficiente de variación

Dunnett (8), con un nivel de significancia de $p=0,05$ para acetilcolinesterasa modificada y CIP450, no se encontraron diferencias significativas. Para las enzimas esterasas inespecíficas, en cambio, los municipios de Sabana de Torres $(0,23)$ y Villavicencio $(0,20)$ presentaron diferencias significativas entre valores de medias de densidad óptica con respecto a la cepa de referencia $(p=0,05)$.

\section{Discusión}

Para el conjunto de las poblaciones colombianas del vector del dengue Ae. aegypti evaluadas en este estudio, se encontró susceptibilidad fisiológica al carbamato propoxur y al organofosforado malatión. Los resultados de susceptibilidad de las poblaciones de adultos de Ae. aegypti al malatión obtenidos en este estudio, coinciden con los estudios anteriores para esta especie en Cuba, Venezuela, Panamá, Perú y Colombia (20-25). Este hallazgo reviste gran importancia, teniendo en cuenta que la aplicación espacial de este insecticida es la medida más utilizada en Colombia para interrumpir la transmisión del dengue durante los brotes o epidemias. De manera particular, si se tienen en cuenta los hallazgos de pérdida de susceptibilidad al piretroide lambdacialotrina para las mismas poblaciones incluidas en este estudio (25), este organofosforado continúa siendo de elección.

Los resultados obtenidos para el insecticida fenitrotión revelaron susceptibilidad en la mayoría de las poblaciones evaluadas $(9 / 13)$, con bioensayos OMS y CDC. Las cuatro poblaciones restantes mostraron porcentajes de mortalidad con OMS entre 14 y $30 \%$ y, por lo tanto, indicativos de resistencia, en contraste con los resultados de $100 \%$ de mortalidad utilizando la metodología CDC. En un estudio con Ae. aegypti en el Valle del Cauca, también se habían encontrado incongruencias para estas dos metodologías cuando se evaluó el insecticida malatión, lo cual se asoció con problemas de calidad en los papeles impregnados OMS (24).

Es de aclarar que, aunque ambas pruebas expresan los resultados en porcentajes de mortalidad, responden a interrogantes distintos. En general, los bioensayos CDC miden el tiempo que tarda el principio activo a una dosis específica en llegar al sitio blanco de acción; entre más tiempo tarde la molécula en actuar en el insecto, se entiende que se puede estar desarrollando un mecanismo fisiológico de desintoxicación en éste (16). En este contexto, la dosis evaluada se expresa en microgramos por mililitro que, para el caso de fenitrotión en este estudio, correspondió a $75 \mu \mathrm{g} /$ $\mathrm{ml}$ por 45 minutos. En los bioensayos OMS, se evalúa la respuesta de las poblaciones naturales de los mosquitos a la dosis de ingrediente activo que debería ser eficaz en condiciones de campo. En este estudio se utilizaron papeles impregnados al 1,0 \% para fenitrotión. Para una misma población natural de Ae. aegypti se espera que, si se encuentra susceptibilidad utilizando las dosis y tiempos diagnósticos del bioensayo $\mathrm{CDC}$, se 
encuentre también susceptibilidad con el bioensayo OMS. Sin embargo, los resultados obtenidos para estas cuatro localidades requieren de verificación.

De acuerdo con la incongruencia en los resultados OMS y CDC para propoxur y fenitrotión, debe tenerse en cuenta que, debido a que los papeles impregnados deben adquirirse en Malasia, único centro autorizado por OMS, pueden existir dificultades durante el proceso de envío y almacenamiento hasta su uso, los que puedan afectar la estabilidad de algunas moléculas insecticidas. Además, los procesos administrativos de importación pueden llegar a incrementar los tiempos entre el envío y el uso de los papeles impregnados, lo que determina en muchos casos que los bioensayos se utilicen cerca a las fechas de vencimiento. En los programas de vigilancia de la resistencia a insecticidas nacionales, es importante establecer si se aplican los bioensayos OMS y CDC para una misma población natural de vectores o si solo se aplica una sola metodología utilizando la cepa de referencia sensible como control positivo.

Para el larvicida temefos, se observó resistencia en el $70 \%$ de las poblaciones evaluadas, lo cual se explica porque este ha sido el insecticida de elección para el tratamiento focal de formas inmaduras del vector en áreas endémicas en Colombia. A pesar de que en Colombia la resistencia de Ae. aegyptial temefos se ha demostrado en diferentes estudios, los registros de resistencia al malatión son escasos $(7,9)$ (González J, Rey G, Olano V, Brochero H. Informe técnico, Ministerio de la Protección Social. 2005; Convenio 043. p. 60). Sin embargo, en un estudio reciente en Brasil, se demostró resistencia a los dos inseciticidas en el nordeste de este país, lo cual se asoció con el uso de organofosforados en estado de larva y de adulto (26). Lo anterior indica que, a pesar de los resultados de sensibilidad para la mayoría de poblaciones de Ae. aegypti de Colombia evaluadas para el malatión, se debe mantener la vigilancia de la resistencia a esta molécula, particularmente si se continúa con su uso en forma sistemática.

Los resultados de las pruebas bioquímicas demostraron comportamientos diferenciales entre comunas pertenecientes a un mismo municipio. Estas diferencias se explican por la heterogeneidad misma de las poblaciones evaluadas, las cuales correspondieron a comunas diferentes de cada municipio. Las comunas consisten en agrupaciones de barrios con características similares socioculturales, demográficas, epidemiológicas y entomológicas para dengue; con base en esta agregación geográfica se establece el riesgo y se focalizan las estrategias de control. Esta circunstancia determina las diferencias en la intensidad y el tiempo durante el cual las poblaciones evaluadas han estado presionadas por los insecticidas utilizados en los programas de control y por las mismas comunidades.

En estudios anteriores se ha asociado el incremento de las enzimas esterasas inespecíficas con resistencia fisiológica a organosfosforados en diferentes especies de insectos, incluido Ae. aegypti (27-30). De manera particular, a las esterasas a y $\beta$ se les atribuye la pérdida de sensibilidad a temefos (31-33). No obstante, se encontró que tres de las cuatro poblaciones de Ae. aegypti correspondientes a Puerto López 1, Bucaramanga 17 y Villavicencio 7 que registraron susceptibilidad fisiológica a este principio activo, presentaron valores superiores al punto de corte para enzimas esterasas inespecíficas, en tanto, otras que mostraron resistencia fisiológica a esta misma molécula, como Girardot 1 y 3 y La Mesa 2, registraron valores por debajo del punto de corte.

Lo anterior puede explicarse porque las pruebas bioquímicas se aplicaron en poblaciones de $A e$. aegypti de individuos adultos y no de larvas, estadio usado para los bioensayos. Algunos autores recomiendan aplicar las pruebas bioquímicas sobre el estado de desarrollo que se pretende controlar, debido a que los mecanismos de resistencia no necesariamente se encuentran en todos los estadios de desarrollo del insecto (34).

Estas diferencias relacionadas con el estado de desarrollo se han confirmado en otros estudios. En Brasil, se encontraron diferencias en la expresión del nivel de las enzimas CIP450 monooxigenasas, relacionadas con el estado de desarrollo (28) y, en Cuba, un estudio en el cual se evaluó la resistencia a malatión en larvas y adultos reveló diferencias en la susceptibilidad para los dos estadios de desarrollo (35). Evidentemente, la expresión genética de la especie se desarrolla en concordancia con el nicho que ocupa, por lo cual la desintoxicación de xenobióticos está determinada por la fisiología del estado de desarrollo en el cual se encuentre el insecto y, de manera particular, para las enzimas esterasas inespecíficas asociadas con procesos naturales de desintoxicación, las cuales también incrementan su expresión con la longevidad de los individuos. 
En este sentido, a pesar de la imposibilidad de establecer una asociación directa entre los resultados de resistencia a temefos encontrados con pruebas biológicas y los resultados de las pruebas bioquímicas, no se puede descartar que las enzimas esterasas inespecíficas constituyan el mecanismo bioquímico responsable de la resistencia a este insecticida, debido a las diferencias de selección de resistencia de las enzimas esterasas inespecíficas entre larvas y adultos. Lo anterior se podría corroborar aplicando pruebas bioquímicas o de sinergias sobre los estadios larvales de las poblaciones de Ae. aegypti que revelaron disminución de la sensibilidad en este estudio.

Para las poblaciones de adultos pertenecientes a La Mesa 1, Villavicencio 5 y 7, Sabana de Torres 1 y 2 y Bucaramanga 17 evaluadas por los autores en otro estudio, se encontró asociación entre las enzimas esterasas inespecíficas elevadas y la resistencia fisiológica a lambdacialotrina (25), por lo que no se podría descartar la posibilidad de encontrar resistencia cruzada entre piretroides y organofosforados, ambos de amplio uso para el control del insecto en estas poblaciones. En larvas de Ae. aegypti de Cuba, se observó resistencia a piretroides por presión con malatión (35) y, en Brasil, se encontró una resistencia fisiológica elevada de adultos a los piretroides y una baja resistencia al temefos (28). La resistencia cruzada a temefos con piretroides como deltametrina y cipermetrina, y con el organofosforado malatión, se comprobó en condiciones controladas de laboratorio (32). En Colombia, estos insecticidas son los autorizados para el control de dengue y de malaria, y también son de amplio uso en agricultura, lo que conlleva a plantear la necesidad implementar esquemas de vigilancia estricta, de rotación de insecticidas y áreas con disminución en la presión de selección con estas moléculas. Teniendo en cuenta esto, se debe considerar que la supresión de la presión con insecticidas puede revertir la resistencia, lo cual se ha corroborado en condiciones controladas de laboratorio (32).

La ausencia de individuos con acetilcolinesterasa modificada en las poblaciones de Ae. aegypti evaluadas en este estudio, concuerda con estudios en los cuales no se ha encontrado este mecanismo asociado con resistencia a organofosforados en esta especie $(32,33)$. Sin embargo, se requiere hacer pruebas bioquímicas para determinar los niveles de acetilcolinesterasa modificada en las poblaciones de larvas, con el propósito de conocer la responsabilidad de este mecanismo en la resistencia al temefos en poblaciones de Colombia. Para Bucaramanga 17, la asociación entre los resultados de las pruebas biológicas y bioquímicas no es muy clara. Esta población reveló susceptibilidad a todos los insecticidas evaluados. Sin embargo, también mostró incrementos significativos en las enzimas CIP450 monooxigenasas, enzimas esterasas inespecíficas y la presencia de una proporción de individuos con acetilcolinesterasa modificada modificada. En este sentido, se puede interpretar que esta población puede potencialmente desarrollar resistencia a insecticidas de cualquier grupo químico, aspecto relevante si se tiene en cuenta que este municipio aporta la mayor carga de casos de dengue clásico y dengue grave para el departamento de Santander $y$, por ende, las poblaciones naturales del mosquito vector son fuerte y frecuentemente presionadas con insecticidas químicos. Es urgente, entonces, ampliar los estudios de susceptibilidad a otras localidades urbanas de Bucaramanga y de los municipios cercanos a su área de influencia.

Las diferencias en el nivel de enzimas obedecen a diferencias locales en cuanto a la presión de selección a partir del control químico implementado para el control del dengue y a la variabilidad genética propia de cada población. La resistencia fisiológica a insecticidas constituye un fenómeno localizado de manera específica en espacio y tiempo, y por consiguiente, las particularidades que presentaron algunas de las poblaciones de $A e$. aegypti evaluadas en este estudio deben tenerse en cuenta para el establecimiento de un programa de vigilancia y prevención de la resistencia. Ante la posibilidad de que exista resistencia cruzada a lambdacialotrina causada por presión de selección por uso continuo de temefos, se sugiere mantener vigilancia sistemática de la resistencia a estas moléculas y disminuir la presión de selección con estos insecticidas en el país, mediante el fortalecimiento de otras medidas de control del vector, como la destrucción de criaderos, el control físico, el uso insecticidas biológicos, la rotación con otras moléculas químicas y, particularmente, mediante la implementación de estrategias de acción-participación de la comunidad que ha mostrado resultados favorables en varios países (36). En este mismo contexto, debe incorporarse a la vigilancia nacional de la resistencia a insecticidas la evaluación de enzimas glutatión S-transferasa, las cuales se han asociado como mecanismo de desintoxicación del DDT (37) y, recientemente, 
como mecanismo de resistencia fisiológica al temefos (32).

Colombia ha descentralizado las actividades de control de la transmisión del dengue, las cuales han sido asumidas por las autoridades municipales de salud. Se sabe que la exposición a dosis subletales de insecticidas químicos induce la expresión de sistemas de desintoxicación fisiológica en las poblaciones naturales de insectos (38), por lo que se requiere también establecer procesos de capacitación regular para los procesos de aplicación de insecticidas, tales como dosis, tamaño de gota y hora de aplicación, entre otros. Evidentemente, los hallazgos de resistencia fisiológica deben complementarse con estudios sobre la genética de las poblaciones naturales del mosquito y la susceptibilidad que presenten a la infección por arbovirus de tipo dengue.

\section{Agradecimientos}

Los autores expresan los agradecimientos a las secretarías de salud de Caquetá, Cundinamarca, Guaviare, Santander y Meta, y al Instituto Nacional de Salud, por el apoyo institucional y la colaboración del personal técnico y profesional en este trabajo.

\section{Conflicto de intereses}

Los autores declaramos que no existen conflictos de intereses en la realización y difusión de este trabajo.

\section{Financiación}

Este trabajo fue financiado por el Instituto Colombiano para el Desarrollo de la Ciencia y la Tecnología "Francisco José de Caldas" Colciencias, código 219-2004 y el Instituto Nacional de Salud.

\section{Referencias}

1. Guzmán M, García G, Kourí G. El dengue y el dengue hemorrágico: prioridades de investigación. Rev Panam Salud Pública. 2006;19:204-15. http://dx.doi.org/10.1590/ S1020-49892006000300015

2. Cáceres-Manrique FM, Vesga-Gómez C, Perea-Florez X, Ruitort M, Talbot Y. Conocimientos, actitudes y prácticas sobre dengue en dos barrios de Bucaramanga, Colombia. Rev Salud Pública. 2009;11:27-38. http://dx.doi.org/10.1590/ S0124-00642009000100004

3. World Health Organization (WHO), Special Programme for Research and Training in Tropical Diseases (TDR). Dengue guidelines for diagnosis, treatment, prevention and control. Geneva: WHO; 2009.

4. Instituto Nacional de Salud, Subdirección de Vigilancia y Control en Salud Pública. Boletín epidemiológico número 52. Bogotá D.C.; Instituto Nacional de Salud: 2010. p. 11.
5. Wenceslao P, Pedroni E. Análisis costo-beneficio del control de vectores en la transmisión potencial de dengue. Rev Panam Salud Pública. 2008;24:113-9. http://dx.doi. org/10.1590/S1020-49892008000800005

6. Mancheno M, Kroeger A, Álvarez G. Manual técnico para el control de malaria, dengue, leishmaniosis y oncocercosis. Bogotá, D.C.: Servi Offset Ltda.; 1998. p. 264.

7. Ocampo C, Salazar M, Mina N, McAllister J, Brogdonc W. Insecticide resistance status of Aedes aegypti in 10 localities in Colombia. ActaTrop. 2011;2611:1-8. http:// dx.doi.org/10.1016/j.actatropica.2011.01.007

8. Busvine J, Coker W. Resistance patterns in DDT resistant Aedes aegypti. Bull World Health Organ. 1958;18:651-6.

9. Maestre R, Rey G, De las Salas J, Vergara C, Santacoloma L, Goenaga S, et al. Susceptibilidad de Aedes aegypti (Diptera: Culicidae) a temefos en Atlántico-Colombia. Revista Colombiana de Entomología. 2009;35:202-5.

10. Mazzari M. Revisión del estado actual de la resistencia en Aedes aegypti a insecticidas utilizados en salud pública. Boletín de la Dirección de Malariología y Saneamiento Ambiental. 2005;35:90-5.

11. Organización Panamericana de la Salud (OPS). Dengue y dengue hemorrágico en las Américas: guías para su prevención y control. Washington, D.C.: Organización Panamericana de la Salud; 1995. p. 108.

12. Organización Mundial de la Salud. Instructions for determining the susceptibility or resistance of mosquitoes larvae to insecticide. WHO/VBC/81.807. Geneva: Organización Mundial de la Salud; 1981.6 p.

13. Fonseca-González I. Estatus de la resistencia a insecticidas de los vectores primarios de malaria y dengue en Antioquia, Chocó, Norte de Santander y Putumayo, Colombia (tesis). Medellín: Universidad de Antioquia; 2008. p. 183.

14. Organización Mundial de la Salud. Instructions for determining the susceptibility or resistance of adult mosquitoes to organoclorine, organophosphorus and carbamate insecticides. Establishment of the base-line. WHO/ VBC/81.805. Geneva: Organización Mundial de la Salud; 1981. p. 8.

15. Brogdon W, Mcallister J. Simplification of adult mosquito bioassays through use of time-mortality determinations in glass bottles. J Am Mosq Control Assoc. 1998;14:159-64.

16. Ciba Geygi. Cómo realizar un bioensayo. Manual de ensayos de campo. Bogotá: Ciba Geygi; 1973. p. 215.

17. Brogdon W. Mosquito protein microssay. Protein determinations from small portions of single-mosquito homogenates. Comp Biochem Physiol B. 1984;79:457-9.

18. Brogdon W. Microassay of acetylcholinesterase activity in small portions of single mosquito homogenates. Comp Biochem Physiol B. 1984;96:339-42.

19. Brogdon W, McAllister J, Vulule J. Heme peroxidase activity measured in single mosquitoes identifies individuals expressing and elevated oxidases for insecticide resistance. J Am Mosq Control Assoc. 1997;13:233-7.

20. Rodríguez MM, Bisset JA, Molina D, Soca L. Malathion resistance in Aedes aegypti and Culex quinquefasciatus after its use in Aedes aegypti control programs. J Am Mosq Control Assoc. 2000;16:324-30. 
21. Molina D, Bisset J, Rodríguez M, González J, Salas $\mathrm{O}$, Barazarte $\mathrm{H}$, et al. Estudio de la susceptibilidad a insecticidas organofosforados y piretroides en cepas de Aedes aegypti (Linn.) de cinco estados de Venezuela. Bol Dir Malariol y San Amb. 1995;35:85-95.

22. Bisset J, Rodríguez M, Cáceres L. Niveles de resistencia y sus mecanismos en 2 cepas de Aedes aegypti de Panamá. Rev Cubana Med Trop. 2003;55:191-5.

23. Valderrama E, González O, Jaramillo G. Evaluación de la susceptibilidad de Aedes aegypti (L) (Diptera: Culicidae) a un insecticida organofosforado y un piretroide en cuatro poblaciones del Valle del Cauca, mediante dos tipos de bioensayos. Boletín del Museo de Entomología de la Universidad del Valle. 2008;9:1-11.

24. Bisset J, Rodríguez M, De Armas Y. Comparación de dos poblaciones de mosquitos Aedes aegypti de Santiago de Cuba con diferente conducta de reposo. Rev Cubana Med Trop. 2005;57:143-50

25. Santacoloma L, Chaves B, Brochero HL. Susceptibilidad de Aedes aegypti a DDT, deltametrina y lambdacialotrina en Colombia. Rev Panam Salud Pública. 2010;27:66-73. http:// dx.doi.org/10.1590/S1020-49892010000100010

26. Macoris M, Andrighetti M, Garbeloto C, Carvalho L, Junior A, Brogdon W. Association of insecticide use and alteration on Aedes aegypti susceptibility status. Mem Inst Oswaldo Cruz. 2007;102:895-900. http://dx.doi.org/10.1590/ S0074-02762007000800001

27. Karunaratne S, Hemingway J. Malathion resistance and prevalence of the malathion carboxylesterase mechanism in populations of mosquito vectors of disease in Sri Lanka. Bull World Health Organ. 2001;79:1060-79. http://dx.doi. org/10.1590/S0042-96862001001100010

28. Macoris M, Andrighetti M, Takaku L, Glasser C, Garbeloto V, Bracco J. Resistance of Aedes aegypti from the state of São Paulo, Brazil, to organophosphates insecticides. Mem Inst Oswaldo Cruz. 2003;98:703-8. http:// dx.doi.org/10.1590/S0074-02762003000500020

29. Rodríguez M, Bisset J, Mila L, Calvo E, Diaz C, Soca L. Niveles de resistencia a insecticidas y sus mecanismos en una cepa de Aedes aegypti de Santiago de Cuba. Rev Cubana Med Trop. 1999;51:83-8.
30. Bisset J, Rodríguez M, Molina D, Díaz C, Soca A. Esterasas elevadas como mecanismo de resistencia a insecticidas organofosforados en cepas de Aedes aegypti Diptera: Culicidae. Rev Cubana Med Trop. 2001;53:37-43.

31. Chávez G, Roldán J, Vargas F. Niveles de resistencia a dos insecticidas en poblaciones de Aedes aegypti (Díptera:Culicidae) del Perú. Revista Colombiana Entomología. 2005;31:75-8.

32. Melo-Santos MA, Varjal-Melo JJ, Araújo AP, Gomes TC, Paiva MH, Regis LN, et al. Resistance to the organophosphate temephos: Mechanisms, evolution and reversion in an Aedes aegypti laboratory strain from Brazil. Acta Trop. 2009;113:180-9. http://dx.doi.org/10.1016/j. actatropica.2009.10.015

33. Rodríguez M, Bisset J, Fernández D, Pérez O. Resistencia a insecticidas en larvas y adultos de Aedes aegypti: prevalencia de la esterasa A4 asociada con la resistencia a temefos. Rev Cubana Med Trop. 2004;56:54-60.

34. Symondson W, Hemingway J. Biochemical and molecular techniques. En: Dent DR, Walton MP, editors. Methods in ecological and agricultural entomology. New York: Cab International; 1997. p. 293-350.

35. Rodríguez M, Bisset J, Mila L, Calvo E, Díaz C, Soca L. Niveles de resistencia a insecticidas y sus mecanismos en una cepa de Aedes aegypti de Santiago de Cuba. Rev Cubana Med Trop. 1999;51:83-8.

36. World Health Organization. Prevention and control of dengue and dengue haemorrhagic fever. Comprehensive guidelines. New Delhi: SEARO, WHO Regional Publications; 1999. p. 134

37. Hemingway J, Hawkes NJ, McCarroll L, Ranson H. The molecular basis of insecticide resistance in mosquitoes. Insect Biochem Mol Biol. 2004;34:653-6. http://dx.doi. org/10.1016/j.ibmb.2004.03.018

38. Poupardin R, Reynaud S, Strode C, Ranson H, Vontas J, David JP. Cross-induction of detoxification genes by environmental xenobiotics and insecticides in the mosquito Aedes aegypti: Impact on larval tolerance to chemical insecticides. Insect Biochem Mol Biol. 2008;38:540-51. http://dx.doi.org/10.1016/j.ibmb.2008.01.004 